\section{Psychological Medicine}

cambridge.org/psm

\section{Original Article}

*These authors contributed equally to this study.

Cite this article: Wang $\mathrm{H}$, Huang $\mathrm{D}$, Huang $\mathrm{H}$ Zhang J, Guo L, Liu Y, Ma H, Geng Q (2022). The psychological impact of COVID-19 pandemic on medical staff in Guangdong, China: a cross-sectional study. Psychological Medicine 52, 884-892. https://doi.org/10.1017/ S0033291720002561

Received: 18 February 2020

Revised: 26 April 2020

Accepted: 2 July 2020

First published online: 6 July 2020

\section{Key words:}

Anxiety; coronavirus disease 2019; COVID-19; medical staff; psychological status

\section{Authors for correspondence:}

Yuting Liu,

E-mail: liuyuting0817@126.com;

Huan Ma,

E-mail: mahuandoctor@163.com;

Qingshan Geng,

E-mail: gengqingshan@gdph.org.cn

(c) The Author(s), 2020. Published by Cambridge University Press. This is an Open Access article, distributed under the terms of the Creative Commons Attribution licence (http://creativecommons.org/licenses/by/4.0/), which permits unrestricted re-use, distribution, and reproduction in any medium, provided the original work is properly cited.

\section{CAMBRIDGE} UNIVERSITY PRESS

\title{
The psychological impact of COVID-19 pandemic on medical staff in Guangdong, China: a cross-sectional study
}

\begin{abstract}
Huajun Wang, ${ }^{1, \star}$, Daozheng Huang ${ }^{2, \star}$, Huigen Huang ${ }^{3}$, Jihui Zhang ${ }^{4}$, Lan Guo ${ }^{5}$, Yuting Liu ${ }^{5}$, Huan $\mathrm{Ma}^{5}$ and Qingshan Geng ${ }^{5}$

${ }^{1}$ Intensive Care Unit, Guangdong Provincial People's Hospital, Guangdong Academy of Medical Sciences, Guangzhou, Guangdong, China; ${ }^{2}$ Department of Critical Care Medicine, Guangdong Provincial People's Hospital, Guangdong Academy of Medical Sciences, Guangdong Provincial Geriatrics Institute, Guangzhou, Guangdong, China; ${ }^{3}$ Guangdong Provincial People's Hospital, Guangdong Academy of Medical Sciences, Guangzhou, Guangdong, China; ${ }^{4}$ Department of Psychiatry, Faculty of Medicine, The Chinese University of Hong Kong, Shatin, NT, Hong Kong SAR, China and ${ }^{5}$ Guangdong Provincial Key Laboratory of Coronary Heart Disease Prevention, Guangdong Cardiovascular Institute, Guangdong Provincial People's Hospital, Guangdong Academy of Medical Sciences, Guangzhou, Guangdong, China
\end{abstract}

\begin{abstract}
Background. During previous pandemic outbreaks, medical staff have reported high levels of psychological distress. The aim of the current study was to report a snapshot of the psychological impact of the coronavirus disease 2019 (COVID-19) pandemic and its correlated factors on medical staff in Guangdong, China.

Methods. On the 2nd and 3rd February 2020, soon after the start of the COVID-19 pandemic, we surveyed medical staff at four hospitals in Guangdong, China, to collect demographic characteristics, Hospital Anxiety and Depression Scale (HADS), Perceived Stress Scale (PSS-14), and Insomnia Severity Index (ISI) scores.

Results. Complete responses were received from 1045 medical staff. Respondents were divided into high- and low-risk groups according to their working environment of contacting with potential or confirmed COVID-19 cases. The proportion of staff with anxiety $(55.4 \% v$. $43.0 \%, p<0.001)$ or depression $(43.6 \% v .36 .8 \%, p=0.028)$ was significantly higher in the high-risk group than the low-risk group. The percentage of staff with severe anxiety was similar in the two groups. Doctors were more susceptible to moderate-to-severe depressive symptoms. The high-risk group had higher levels of clinical insomnia $(13.5 \% v .8 .5 \%, p=$ $0.011)$ and were more likely to be in the upper quartile for stress symptoms $(24.7 \% v$. $19.3 \%, p=0.037$ ) than the low-risk group. Additionally, work experience negatively correlated with insomnia symptoms.

Conclusions. It is important for hospitals and authorities to protect both the physical and psychological health of medical staff during times of pandemic, even those with a low exposure risk.
\end{abstract}

\section{Introduction}

In late December 2019, clusters of pneumonia cases with unknown cause emerged in Wuhan, China. Sequencing of lower respiratory lavage fluid indicated that the disease was caused by a novel coronavirus in humans that was subsequently named 2019 novel coronavirus (2019-nCoV) by the World Health Organization (WHO, 2020a). The novel coronavirus is thought to be a relative of the deadly severe acute respiratory syndrome (SARS) and Middle East respiratory syndrome viruses. These coronaviruses are all characterised by flu-like symptoms, including fever, cough, and chills - they can also cause severe respiratory illness and death (WHO, 2020a). During Spring Festival in China, hundreds of thousands of people left Wuhan, often via public transport, potentially carrying the virus with them. As a result, the virus is now rapidly spreading worldwide.

On the 31st January 2020, the WHO declared the Coronavirus Disease 2019 (COVID-19) outbreak in China constituted a Public Health Emergency of International Concern (PHEIC) (WHO, 2020b). By the 1st February 2020, 14380 cases of COVID-19 were confirmed nationwide by the Chinese government (including medical staff), and another 19544 cases were suspected (National Health Commission, 2020). Shortly after, hundreds of cases were confirmed in Thailand, Japan, South Korea, German, and the United States of America (WHO, 2020c).

Because of the urgency of the situation, most current research has mainly focused on the clinical manifestations and epidemiology of COVID-19. The psychological impact and distress associated with COVID-19 is largely neglected. Dealing with a highly infectious disease outbreak entails a risk of infection, psychological stress, and emotional challenges for all those 
involved. A study of the 2003 SARS outbreak showed medical staff in Toronto, Canada, suffered from high levels of psychological stress while treating patients (Maunder, 2004). About $10 \%$ of medical staff in Beijing have experienced high levels of posttraumatic stress symptoms since the SARS outbreak (Wu et al., 2009). High burdens of stress among medical staff were also documented during the Ebola outbreak in 2014 (Lehmann et al., 2016). Furthermore, during the H1N1 influenza pandemic in Japan, medical staff in high-risk work environments felt more anxious and more exhausted than those in lower risk environments (Matsuishi et al., 2012). Other studies have also shown an association between negative life-events (including occupational stress) and higher levels of anxiety and psychological distress (Shigemura, Tanigawa, \& Nomura, 2012; Weinberg \& Creed, 2000).

To date, there are few studies reporting on the psychological impact of COVID-19 in a large population. Lai and colleagues have found a considerable proportion of medical staff who worked around patients with COVID-19 in several cities around China to have symptoms of depression, anxiety, insomnia, and distress (Lai et al., 2020). Medical staff in high risk departments are potentially more susceptible to psychological disorders ( $\mathrm{Lu}$, Wang, Lin, \& Li, 2020), and compared with many other occupations, a high proportion of medical staff report of poor sleep quality (Huang \& Zhao, 2020). Sleep problems and stress are closely related to the development of depression and anxiety (Cox \& Olatunji, 2020; Ford \& Kamerow, 1989; Kendler, Thornton, \& Gardner, 2000).

Medical staff nearly always receive intensive infection control training before dealing with highly infectious patients; however, their safety can never be guaranteed. In addition to their fears of personal contamination, medical staff may have to cope with other stresses, including the deaths of colleagues, threats to their family's safety, and working excessive hours. Some may also suffer from stigmatisation and violence targeted against health professionals (Lancet, 2020).

Given the possibility of future pandemics, the psychological impact of the current COVID-19 pandemic on medical staff should be evaluated. This study reports the immediate stress and psychological impact of COVID-19 on medical staff in several seriously affected hospitals in Guangdong province, China, around 1 month after the first Chinese case was reported. The number of confirmed cases in Guangdong province has increased to 604 as of 2nd February 2020, with the second rank in China (Health Commission, 2020).

\section{Methods}

\section{Study sample}

Medical staff (doctors, nurses, and auxiliary staff) in Guangdong Provincial People's Hospital, Luoding People's Hospital, Yingde People's Hospital, and Huizhou Sixth People's Hospital, all in Guangdong province, completed electronic questionnaires between the 2nd and 3rd February 2020. The questionnaire was distributed to all staff who worked in the fever clinic, emergency department, intensive care unit (ICU), infectious disease department, and three to four medical wards or auxiliary departments by administrators (e.g. department head nurse) at each hospital via WeChat ${ }^{\mathrm{TM}}$ (Tencent Inc., Shenzhen) work groups.

Participation was strictly voluntary and all responses were anonymous. The response rate for the questionnaire was calculated according to the total number of individuals in each WeChat ${ }^{\mathrm{TM}}$ group.

\section{Content of questionnaire}

A cover note, stating that the purpose of the survey was to examine the mental health status of the medical staff during the COVID-19 pandemic, was distributed with the questionnaire. The note also stated that the results of the survey would be published, and that the respondent's answers would remain anonymous. The survey consisted of questions on demographic characteristics and psychological status.

The surveyed demographic characteristics included gender, education, job, years of employment in the current role, working department, and work environment during the COVID-19 pandemic. Jobs were categorised as either medical doctor, nurse, or auxiliary staff (radiological technologists, clinical laboratory technicians, pharmacists, dieticians, physical therapists, office workers, or clinical clerks). Work environment was categorised as high-risk (direct contact with a confirmed or suspected case of COVID-19) or low-risk (no direct contact).

To assess the staff's psychological distress, the Hospital Anxiety and Depression Scale (HADS), Perceived Stress Scale (PSS-14), and Insomnia Severity Index (ISI) were used.

The HADS was devised 30 years ago by Zigmond and Snaith (1983) to measure anxiety and depression in a general medical population (see online Additional File 1). Many studies have confirmed the validity of the HADS in the setting for which it was designed. The HADS consists of 14 items, divided in two seven-item subscales measuring anxiety (HADS-A) and depression (HADS-D). Items are scored on a 4-point Likert scale ranging from 0 to 3 , providing total subscale scores from 0 to 21 . A score $\leqslant 7$ corresponds to 'no depression or anxiety', a score of $8-10$ is 'minor depression/anxiety', a score of 11-15 is 'moderate depression/ anxiety', and a score $\geqslant 16$ is defined as 'severe depression/anxiety'.

Perceived stress status was evaluated with the Chinese version of PSS-14 (see online Additional File 2) (Cohen, Kamarck, \& Mermelstein, 1983), seven positive and seven negative, which has been validated in Chinese cardiac patients (Leung, Lam, \& Chan, 2010). The PSS was developed to appraise whether respondents considered their life to be unpredictable, uncontrollable, or overloaded. Each item was scored on a 5-point Likert scale ranging from $0=$ never to $4=$ very often. PSS scores were obtained by reversing the scores on the seven positive items (e.g. $0=4,1=$ $3,2=2,3=1$, and $4=0$ ) (items 4, 5, 6, 7, 9, 10, and 13), and then summing across all scale items. A higher score represents a higher stress level and also an increased likelihood that environmental demands exceed an individual's ability to cope. As there is a lack of studies proposing a standard cut-off score to diagnose or grade stress, we categorised the PSS scores into four quartiles. The lower quartile includes scores $<20$, the second quartile ranges from 20 to 26 , the third quartile ranges from 27 to 29 , and the upper quartile includes scores that are $>29$ out of a possible 56 .

The ISI is a commonly used seven-item psychometrically validated measure used to perceive the severity of insomnia symptoms and associated functional impairment. A 5-point Likert scale $(0=$ not at all, $4=$ extremely) is used to rate each of these items, yielding to a total score ranging from 0 to 28 , with scores of $0-7$ indicating absence of insomnia, 8-14 indicating sub-threshold insomnia symptoms, 15-21 indicating moderate insomnia, and 22-28 indicating severe insomnia (Morin, Beaulieu-Bonneau, LeBlanc, \& Savard, 2005). The Chinese version of the ISI questionnaire was used in this study (see online Additional File 3). This version has good internal consistency, test-re-test reliability, and convergent validity in Chinese population (Chung, Kan, \& Yeung, 2011; Wong et al., 2017). 


\section{Statistical analysis}

All analyses were performed using SPSS (version 11.0, SPSS Inc., Chicago). Descriptive statistics for the HADS, PSS, and ISI scores are presented as medians and interquartile ranges. The HADS, PSS, and ISI scores were converted from continuous variables to dichotomous variables based on their cut-offs as mentioned above: HADS divided as 'no-minor anxiety/depression (HADS $\leqslant 10)$ ' and 'moderate-severe anxiety/depression ( $10<$ HADS)', PSS divided as ' $1-3$ quartile (PSS $\leqslant 29$ )' and 'highest quartile $(29<$ PSS)' and ISI score divided as 'no-subthreshold insomnia (ISI $\leqslant 14)$ ' and 'clinical insomnia (14<ISI)'. Normality was tested by the Shapiro-Wilk test. Because HADS, PSS, and ISI scores tend to have skewed distributions, we used Mann-Whitney $U$ test to assess differences in HADS, PSS, and ISI scores by work environment. Categorical variables were expressed as percentages and analysed using Pearson's $\chi^{2}$ test. Univariate comparisons were conducted by univariate logistic regression to assess variables associated with the degree of anxiety and depression, stress status, or severity of insomnia. Multivariate logistic regression analysis was employed to assess the factors most closely associated with staff's degree of anxiety and depression severity, stress status, and sleep disturbances. We treated groupings based on HADS, PSS, and ISI scores as dependent variables. Demographics and variables significantly associated with dependent variables in univariate analyses were treated as independent variables. Spearman correlation analysis was used to identify correlations among HADS, PSS and ISI in all subjects. All statistical tests were twosided and $p<0.05$ was considered significant.

\section{Results}

The questionnaire received a total of 1049 responses. The response rate was $80.1 \%$ from the fever clinic, emergency department, ICU, and infectious disease departments, and 70.3\% from the wards/auxiliary departments. Of the responses, four were excluded due to $\geqslant 1$ missing answers, leaving 1045 questionnaires $(99.6 \%)$ for analysis.

\section{Sample characteristics}

Table 1 presents the respondents' demographic and professional characteristics, along with their scores and classifications on psychological status measures. Most respondents were female (85.8\%) with an education level of 'college' (56.4\%). About $74 \%$ of respondents were nursing staff. At most, $30.9 \%$ of respondents worked in the front-line departments (such as the fever clinic, emergency department, ICU, or infectious disease department). However, 401/1045 respondents (38.4\%) said that they had been in direct contact with a patient confirmed, or suspected to have, COVID-19. This indicates that there are many staff in addition to those listed above departments, who are at risk during a pandemic.

\section{Factors associated with medical staff's anxiety and depression severity}

Of the $499(47.8 \%)$ respondents who presented HADS-A scores $>7,209(20.0 \%$ of the entire sample) presented scores $>10$, indicative of moderate to severe anxiety. Among all respondents, 412 (39.4\%) presented with depression and 142 (13.6\%) with moderate to severe depression. Higher HADS scores were found among
Table 1. Sample characteristics and psychological status $(N=1045)$

\begin{tabular}{|c|c|}
\hline Demographics and psychological status & \\
\hline \multicolumn{2}{|l|}{ Gender } \\
\hline Male & $148(14.2)$ \\
\hline Female & $897(85.8)$ \\
\hline \multicolumn{2}{|l|}{ Education } \\
\hline Junior college & $411(39.3)$ \\
\hline College & $589(56.4)$ \\
\hline Master and above & $45(4.3)$ \\
\hline \multicolumn{2}{|l|}{ Job } \\
\hline Doctor & $149(14.3)$ \\
\hline Nurse & $773(74.0)$ \\
\hline Others & $123(11.7)$ \\
\hline \multicolumn{2}{|l|}{ Years of working } \\
\hline$<1$ year & $81(7.8)$ \\
\hline $2-3$ years & $160(15.3)$ \\
\hline $4-5$ years & $121(11.6)$ \\
\hline$>5$ years & $683(65.3)$ \\
\hline \multicolumn{2}{|l|}{ Department (multiple choices) } \\
\hline Fever clinic & $76(7.3)$ \\
\hline Emergency & $123(11.8)$ \\
\hline $\mathrm{ICU}$ & $45(4.3)$ \\
\hline Infections department & $78(7.5)$ \\
\hline Others & $772(73.9)$ \\
\hline \multicolumn{2}{|l|}{ Work environment } \\
\hline High risk & $401(38.4)$ \\
\hline Low risk & $644(61.6)$ \\
\hline \multicolumn{2}{|l|}{ Psychological distress } \\
\hline HADS-A score & $7(5-10)$ \\
\hline No-minor anxiety (HADS-A $\leqslant 10$ ) & $836(80.0)$ \\
\hline Moderate-severe anxiety (HADS-A > 10) & $209(20.0)$ \\
\hline HADS-D score & $6(3-9)$ \\
\hline No-minor depression (HADS-D $\leqslant 10$ ) & $903(86.4)$ \\
\hline Moderate-severe depression (HADS-D > 10) & $142(13.6)$ \\
\hline PSS score & $26(19.5-29)$ \\
\hline ISI score & $7(5-12)$ \\
\hline No-subthreshold insomnia (ISI $\leqslant 14$ ) & $936(89.6)$ \\
\hline Clinical insomnia (ISI > 14) & $109(10.4)$ \\
\hline
\end{tabular}

ICU, intensive care unit; HADS-A, Hospital Anxiety and Depression Scale-Anxiety; HADS-D, Hospital Anxiety and Depression Scale-Depression; PSS, Perceived Stress Scale; ISI, Insomnia Severity Index.

Values are given as $N(\%)$, continuous variables as score are given as medians and interquartile ranges; jobs classified as medical doctor, nurse, or auxiliary staff (pharmacists, dieticians, physical therapists, office workers, or clinical clerks).

staff in the high-risk grouping, indicating a higher average level of anxiety and depression (HADS-A: 8 [5-10] v. 7 [4-9], $p<0.001$; HADS-D: 7 [4-9] v. 6 [3-9], $p=0.001)$. A higher proportion of staff had anxiety or depression in the high-risk group than in 
Table 2. Variables associated with the hospital staff's anxiety severity $(N=1045)$

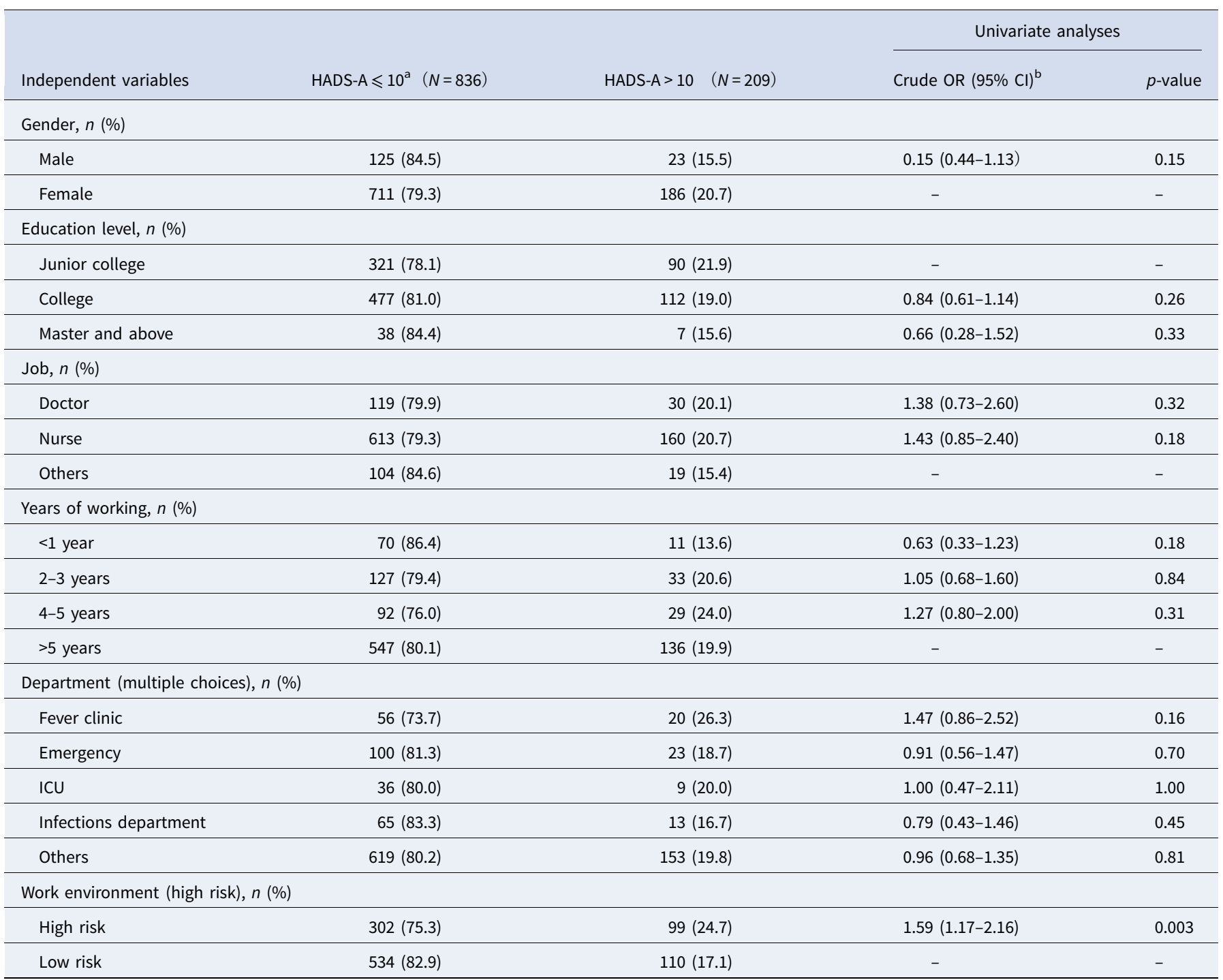

HADS-A, Hospital Anxiety and Depression Scale-Anxiety; ICU, intensive care unit.

${ }^{\mathrm{a} A s}$ reference.

bodds ratios $(95 \% \mathrm{Cl})$.

the low-risk group $(55.4 \%$ v. $43.0 \%, p<0.001 ; 43.6 \%$ v. $36.8 \%, p=$ $0.028)$, while the percentage with severe anxiety was similar $(3.0 \%$ v. $1.6 \%, p=0.115)$.

Univariate analyses (Tables 2 and 3 ) revealed a few variables to be statistically associated with the medical staff's anxiety and depression severity. Subsequent binary multivariate logistic regression analysis showed the odds of being assessed with moderate or severe anxiety $(10<$ HADS-A) (rather than no anxiety or minor anxiety, HADS-A $\leqslant 10$ ) was 1.59 times [95\% confidence interval (CI) 1.17-2.16, $p=0.003$ ]; and the odds of being assessed with moderate or severe depression $(10<$ HADS-D) (rather than no depression or minor depression, HADS-D $\leqslant 10)$ was 1.48 times (95\% CI 1.03-2.11, $p=0.033$ ) higher in the high-risk group compared to the low risk group, after adjustment. The odds of being assessed with moderate or severe depression (rather than no-minor depression) was 2.15 times (95\% CI 1.02-4.56, $p=0.045$ ) greater among doctors compared to any other position; however, this difference was insignificant after adjustment (odds $2.11 ; 95 \%$ CI $0.96-4.64, p=0.065$ ).

\section{Factors associated with medical staff's perceived level of stress}

Binary logistic regression analysis revealed that work environment variables were significantly and independently associated with medical staff's perceived level of stress (Table 4). The odds of being assessed with upper quartile for stress was 1.4 times $(95 \%$ CI 1.02-1.86, $p=0.037$ ) higher in the high-risk group compared with the low-risk group. Furthermore, the proportion of staff in the high-risk group with upper quartile stress was higher than that in the low-risk group $(24.7 \% v .19 .3 \%, p=0.037)$. The average level of perceived stress status was also significantly higher in the high-risk group [PSS: 27 (20-29) v. 25.5 (19-28.75)], with a difference of $1.5(p=0.015)$ between groups.

\section{Factors associated with medical staff's sleep status}

Nearly half $(49.9 \%)$ of the respondents reported insomnia symptoms (ISI $\geqslant 8)$. In total, $109(10.4 \%)$ respondents had a clinical sleep disorders and presented with ISI scores $\geqslant 15$. The proportion 
Table 3. Variables associated with the hospital staff's depression severity $(N=1045)$

\begin{tabular}{|c|c|c|c|c|c|c|}
\hline \multirow[b]{2}{*}{ Independent variables } & \multirow[b]{2}{*}{$\begin{array}{l}\text { HADS-D } \leqslant 10^{a} \\
\quad(N=903)\end{array}$} & \multirow[b]{2}{*}{$\begin{array}{l}\text { HADS-D > } 10 \\
\quad(N=142)\end{array}$} & \multicolumn{2}{|c|}{ Univariate analyses } & \multicolumn{2}{|c|}{ Logistic regression analysis $^{b}$} \\
\hline & & & Crude OR $(95 \% \mathrm{CI})^{\mathrm{c}}$ & $p$-value & Adjusted OR $(95 \% \mathrm{Cl})^{\mathrm{c}}$ & $p$-value \\
\hline \multicolumn{7}{|l|}{ Gender, $n(\%)$} \\
\hline Male & $125(84.5)$ & $23(15.5)$ & $1.20(0.74-1.95)$ & 0.46 & $1.07(0.57-2.01)$ & 0.84 \\
\hline Female & $778(86.7)$ & $119(13.3)$ & - & - & - & - \\
\hline \multicolumn{7}{|l|}{ Education level, $n(\%)$} \\
\hline Junior college & $353(85.9)$ & $58(14.1)$ & - & - & & \\
\hline College & $511(86.8)$ & $78(13.2)$ & $0.93(0.64-1.34)$ & 0.69 & & \\
\hline Master and above & $39(86.7)$ & $6(13.3)$ & $0.94(0.38-2.31)$ & 0.89 & & \\
\hline \multicolumn{7}{|l|}{ Job, $n(\%)$} \\
\hline Doctor & $123(82.6)$ & $26(17.4)$ & $2.15(1.02-4.56)$ & 0.05 & $2.11(0.96-4.64)$ & 0.07 \\
\hline Nurse & $668(86.4)$ & $105(13.6)$ & $1.60(0.83-3.07)$ & 0.16 & $1.66(0.85-3.24)$ & 0.14 \\
\hline Others & $112(91.1)$ & $11(8.9)$ & - & - & - & - \\
\hline \multicolumn{7}{|l|}{ Years of working, $n(\%)$} \\
\hline$<1$ year & $71(87.7)$ & $10(12.3)$ & $0.84(0.42-1.69)$ & 0.63 & & \\
\hline $2-3$ years & $144(90.0)$ & $16(10.0)$ & $0.66(0.60-1.13)$ & 0.15 & & \\
\hline $4-5$ years & $103(85.1)$ & $18(14.9)$ & $1.04(0.61-1.80)$ & 0.88 & & \\
\hline$>5$ years & $585(85.7)$ & $98(14.3)$ & - & - & & \\
\hline \multicolumn{7}{|c|}{ Department (multiple choices), $n(\%)$} \\
\hline Fever clinic & $62(81.6)$ & $14(18.4)$ & $1.48(0.82-2.73)$ & 0.20 & & \\
\hline Emergency & $101(82.1)$ & $22(17.9)$ & $1.46(0.88-2.40)$ & 0.14 & & \\
\hline ICU & $38(84.4)$ & $7(15.6)$ & $1.18(0.52-2.70)$ & 0.69 & & \\
\hline Infections department & $66(84.6)$ & $12(15.4)$ & $1.17(0.62-2.23)$ & 0.63 & & \\
\hline Others & $676(87.6)$ & $96(12.4)$ & $0.70(0.48-1.03)$ & 0.07 & & \\
\hline \multicolumn{7}{|c|}{ Work environment (high risk), $n(\%)$} \\
\hline High risk & $335(83.5)$ & $66(16.5)$ & $1.47(1.03-2.10)$ & 0.033 & $1.48(1.03-2.11)$ & 0.033 \\
\hline Low risk & $568(88.2)$ & $76(11.8)$ & - & - & - & - \\
\hline
\end{tabular}

HADS-D, Hospital Anxiety and Depression Scale-Depression; ICU, intensive care unit.

${ }^{\mathrm{a} A s}$ reference.

'Multivariate logistic regression analysis with dependent variable and independent variables 'gender and the statistically significant variables of the univariate comparisons'.

'Odds ratios $(95 \% \mathrm{Cl})$.

of staff in the high-risk group with insomnia symptoms (56.6\%) and clinical insomnia (13.5\%) was higher than that of the low-risk group [45.8\% $(p=0.001)$ and $8.5 \%(p=0.011)$, respectively]. Median ISI scores were also higher [9 (6-13) v. 7 (4-12), $p<$ $0.001]$. The odds of being assessed with a higher degree of insomnia was 1.60 times $(95 \%$ CI $1.07-2.40, p=0.023)$ greater in the high-risk group than the low-risk group. The odds of being assessed with a higher degree of insomnia were 1.88 times $(95 \%$ CI 1.09-3.26, $p=0.023$ ) greater among staff with fewer years of employment in the current role, compared to those with a longer employment. Staff with a higher education level also had a lower chance of suffering from insomnia ( $p=0.027$; Table 5).

\section{The relationships between the HADS, PSS, and ISI of the medical staff}

There was a significant and positive correlation between HADS-A and HAS-D scores $\left(r_{\mathrm{s}}=0.69, p<0.001\right)$, HADS-A and PSS scores $\left(r_{\mathrm{s}}=0.63, p<0.001\right)$, and HADS-A and ISI scores $\left(r_{\mathrm{s}}=0.48, p<\right.$ $0.001)$. There was also a significant and positive correlation between the HADS-D and PSS scores $\left(r_{\mathrm{s}}=0.65, p<0.001\right)$, HADS-D and the ISI scores $\left(r_{\mathrm{s}}=0.42, p<0.001\right)$. And there was a significant and positive correlation between PSS and ISI scores $\left(r_{\mathrm{s}}=0.44, p<0.001\right)$.

\section{Discussion}

The objective of this study was to report on the psychological impact of the COVID-19 pandemic on medical staff in the Guangdong province of China using a cross-sectional survey conducted at four large hospitals. To our knowledge, this is one of the earliest evaluations of psychological distress in medical staff treating COVID-19 patients (Huang \& Zhao, 2020; Lai et al., 2020; Tang et al., 2020; Xiao, Zhang, Kong, Li, \& Yang, 2020). The results of the current study show that in early February 2020, when there were significant public and medical staff concerns 
Table 4. Variables associated with the hospital staff's perceived stress level $(N=1045)$

\begin{tabular}{|c|c|c|c|c|}
\hline \multirow[b]{2}{*}{ Independent variables } & \multirow[b]{2}{*}{$\mathrm{PSS} \leqslant 29^{\mathrm{a}}(N=822)$} & \multirow[b]{2}{*}{ PSS $>29(N=223)$} & \multicolumn{2}{|c|}{ Univariate analyses } \\
\hline & & & Crude OR $(95 \% \mathrm{Cl})^{\mathrm{b}}$ & $p$-value \\
\hline Male & $123(83.1)$ & $25(16.9)$ & $0.72(0.45-1.13)$ & 0.15 \\
\hline Female & $699(77.9)$ & $198(22.1)$ & - & - \\
\hline Junior college & $311(75.7)$ & $100(24.3)$ & - & - \\
\hline College & $474(80.5)$ & $115(19.5)$ & $0.76(0.56-1.02)$ & 0.07 \\
\hline Master and above & $37(82.2)$ & $8(17.8)$ & $0.67(0.30-1.49)$ & 0.33 \\
\hline \multicolumn{5}{|l|}{ Job, $n(\%)$} \\
\hline \multicolumn{5}{|l|}{ Years of working, $n(\%)$} \\
\hline$<1$ year & $64(79.0)$ & $17(21.0)$ & $1.03(0.59-1.82)$ & 0.92 \\
\hline $2-3$ years & $123(76.9)$ & $37(23.1)$ & $1.17(0.77-1.76)$ & 0.46 \\
\hline $4-5$ years & $92(76.0)$ & $29(24.0)$ & $1.22(0.77-1.93)$ & 0.39 \\
\hline$>5$ years & $543(79.5)$ & $140(20.5)$ & - & - \\
\hline \multicolumn{5}{|c|}{ Department (multiple choices), $n(\%)$} \\
\hline Fever clinic & $54(71.1)$ & $22(28.9)$ & $0.64(0.38-1.08)$ & 0.09 \\
\hline Emergency & $94(76.4)$ & $29(23.6)$ & $0.86(0.55-1.35)$ & 0.52 \\
\hline ICU & $34(75.6)$ & $11(24.4)$ & $0.83(0.41-1.67)$ & 0.60 \\
\hline
\end{tabular}

PSS, Perceived Stress Scale; ICU, intensive care unit.

${ }^{a}$ As reference.

bodds ratios $(95 \% \mathrm{Cl})$.

about a novel infectious disease outbreak, $40-50 \%$ of the surveyed medical staff had anxiety or depressive symptoms, and nearly half reported insomnia symptoms.

A significant proportion (38.4\%) of medical staff had already been in contact with $\geqslant 1$ confirmed or suspected case of COVID-19 at the time of the survey, and this group did not only comprise staff from the anticipated 'front-line' departments. Staff classified as having a lower risk of infection reported a lower average level of anxiety, depression, perceived stress status, and insomnia severity than those in the high-risk group; however, they had a similar incidence of severe anxiety. Interestingly, doctors were particularly susceptible to moderate-severe depression. Scores for anxiety (HADS-A), depression (HADS-D), stress (PSS), and insomnia (ISI) were all significantly correlated.

Medical staff are known to experience significant stress during infectious epidemics (Lehmann et al., 2016; Lin \& Li, 2020; Matsuishi et al., 2012; Maunder, 2004; Wu et al., 2009). Reports of the psychological impact of SARS indicated that high levels of distress were common among medical staff in Toronto
(Maunder et al., 2004). Our current study found a significant percentage of medical staff suffered from mood disorder symptoms, including anxiety and depression (47.8\% and $39.4 \%)$, during the COVID-19 influenza pandemic in Guangdong province, mirroring that seen in previous studies conducted in the Fujian province and the regions outside Hubei province (35-40\%) (Huang \& Zhao, 2020; Lai et al., 2020). However, it seems that the prevalence of anxiety and depression among medical staff in Wuhan (the epicentre of the current outbreak) was even higher (about 50-60\%) (Lai et al., 2020). Together, these findings suggest that mental health burden among medical staff during the COVID-19 pandemic is highly correlated with the severity of the pandemic at their workplace.

Close person-to-person contact seems to be the primary mode of transmission for COVID-19 (Li et al., 2020). It is believed COVID-19 is transmitted most readily by respiratory droplets, but the virus also can spread when people touch a surface or object contaminated with infectious droplets and then touch their mouth, nose, or eyes (Lin \& Li, 2020). During our study, 
Table 5. Variables associated with the hospital staff's sleep status $(N=1045)$

\begin{tabular}{|c|c|c|c|c|c|c|}
\hline \multirow[b]{2}{*}{ Independent variables } & \multirow[b]{2}{*}{$|S| \leqslant 14^{a}(N=936)$} & \multirow[b]{2}{*}{$|S|>14(N=109)$} & \multicolumn{2}{|c|}{ Univariate analyses } & \multicolumn{2}{|c|}{ Logistic regression analysis $^{\mathrm{b}}$} \\
\hline & & & Crude OR $(95 \% \mathrm{CI})^{\mathrm{b}}$ & $p$-value & Adjusted OR $(95 \% \mathrm{CI})^{c}$ & $p$-value \\
\hline \multicolumn{7}{|l|}{ Gender, $n(\%)$} \\
\hline Male & $133(89.9)$ & $15(10.1)$ & $0.96(0.54-1.71)$ & 0.90 & $0.94(0.51-1.74)$ & 0.85 \\
\hline Female & $803(89.5)$ & $94(10.5)$ & - & - & - & - \\
\hline Junior college & $357(86.9)$ & $54(13.1)$ & - & - & - & - \\
\hline College & $541(91.9)$ & $48(8.1)$ & $0.59(0.39-0.89)$ & 0.011 & $0.61(0.39-0.94)$ & 0.027 \\
\hline Master and above & $38(84.4)$ & $7(15.6)$ & $1.22(0.52-2.87)$ & 0.65 & & \\
\hline \multicolumn{7}{|l|}{ Job, $n(\%)$} \\
\hline \multicolumn{7}{|l|}{ Years of working, $n(\%)$} \\
\hline$<1$ year & $74(91.4)$ & $7(8.6)$ & $0.95(0.42-2.15)$ & 0.90 & & \\
\hline $2-3$ years & $141(88.1)$ & $19(11.9)$ & $1.35(0.78-2.33)$ & 0.28 & & \\
\hline $4-5$ years & $100(82.6)$ & $21(17.4)$ & $2.10(1.23-3.60)$ & 0.007 & $1.88(1.09-3.26)$ & 0.023 \\
\hline$>5$ years & $621(90.9)$ & $62(9.1)$ & - & - & - & - \\
\hline \multicolumn{7}{|c|}{ Department (multiple choices), $n(\%)$} \\
\hline Fever clinic & $66(86.8)$ & $10(13.2)$ & $1.33(0.66-2.67)$ & 0.42 & & \\
\hline Emergency & $104(84.6)$ & $19(15.4)$ & $1.69(0.99-2.88)$ & 0.06 & & \\
\hline ICU & $39(86.7)$ & $6(13.3)$ & $1.34(0.55-3.24)$ & 0.52 & & \\
\hline \multicolumn{7}{|l|}{ Work environment, $n(\%)$} \\
\hline
\end{tabular}

ISI, Insomnia Severity Index; ICU, intensive care unit.

${ }^{\mathrm{a} A s}$ reference.

'Multivariate logistic regression analysis with dependent variable and independent variables 'gender and the statistically significant variables of the univariate comparisons'.

codds ratios $(95 \% \mathrm{Cl})$.

doctors and nurses were being equipped with full-body protective gear to be exchanged every $4-6 \mathrm{~h}$. To avoid becoming infected when removing the protective equipment, the staff could not eat, drink, or use the bathroom during their working hours. Combining these working conditions with the requirement of staff in isolation wards to have frequent and close patient contact, it is easy to envisage that staff could easily grow mentally and physically exhausted. We suggest that this type of working environment may predispose staff to anxious and depressive emotional disturbances, as well as somatic symptoms.

Nearly half of the respondents in our survey self-reported insomnia, and the perceived stress status and insomnia severity was higher in staff in the high-risk grouping (who had potential or direct contact with infectious patients). A similar situation has previously been noted in research by Chen et al. (2006), and Maunder, Hunter, Vincent, Bennett, and Mazzulli (2003), where medical staff exhibited poor sleep quality during the SARS outbreak. We found that staff with more experience in their role had, on average, lower levels of insomnia (negative correlation); possibly because they were more accustomed to the relevant occupational hazards and had been able to develop resilience to high-stress and high-intensity working conditions. A previous study similarly demonstrated that nurses with less experience are more likely to experience sleeplessness during a highly infectious work situation than those with more experience (Khatony, Zakiei, Khazaie, Rezaei, \& Janatolmakan, 2020).

When faced with a pandemic, not only the conventional frontline staff (in departments such as the fever clinic, emergency department, ICU, or infections disease department) might have a high risk of exposure - others might do too. In our study, around a quarter of the high-risk grouping (which was based on contact) consisted of staff working in other departments. We found that staff in the high-risk group had a similar chance of having severe anxiety than those in the low-risk group. These results are similar to those of Chen, $\mathrm{Wu}$, Yang, and Yen (2005) in Taiwan, China. Additionally, anxiety and depression 
dimensions measuring by General Severity Index during the Ebola outbreak showed no differences between high and low exposures risk staff (Ji, Ji, Duan, Li, \& Duan, 2017). There are several possible explanations for this result. Firstly, exposure to high-intensity and high-risk work settings (such as ICUs and emergency department work), and direct exposure to infected patients, have been shown not to be the primary determinants of adverse psychological outcomes (Maunder et al., 2003); rather, the resilience of medical staff who choose this type of work was. Secondly, a high level of personal protection training, professional experience, and good social support both before and during the outbreak might have buffered the average severity of symptoms among staff in the high-risk group (Dyregrov, Kristoffersen, \& Gjestad, 1996; Weisæth, 2007). Additionally, while the fear around the potential infection (and consequences) applies to all staff, and the actual risk might be higher in those with increased exposure, staff in the low-risk group may also have concerns about understaffing/overworking etc., and their lack of active control around the situation. The interpersonal isolation and stigmatisation from the society might make it worse. A few news reports had reported that some communities claim to prevent medical staff from going home for safety reason.

Our findings show a positive correlation between the level of anxiety and depression with both insomnia, and stress perceived level, among medical staff working during the recent COVID-19 pandemic. This is similar to the previous study of Xiao et al. (2020). There is growing evidence that sleep problems are observed in mood disorders such as depression and anxiety (Cox \& Olatunji, 2020; Ford \& Kamerow, 1989), and that the persistence of stress or trauma can lead to the development of stress-induced illnesses such as depression and anxiety (Kendler et al., 2000). These findings may relate to chronic sensitivity of the hypothalamic-pituitary-adrenocortical axis and chronic overactivation of the sympathetic-adrenal-medulla system; the two main biological mechanisms involved in the reaction to stress and also the pathophysiology of sleep disturbances (Meerlo, Sgoifo, \& Suchecki, 2008). It has been suggested that social support (such as a wide social network) could reduce anxiety and stress, improve self-efficacy, and indirectly improve sleep quality (Xiao et al., 2020). These findings may provide some ideas for ways to prevent and reduce the psychological impact of infectious outbreaks in the future.

The main methodological limitation of the current study was potential sampling bias that may have overestimated the prevalence of psychological symptoms. This is because respondents in mental distress may have been more interested and willing to complete the questionnaire, and the cover note explaining the purpose of the survey may have skewed participant responses and study results. The second potential limitation is that our findings might not be generalisable to other healthcare settings or time periods within the current COVID-19 pandemic. This study represents a snapshot of the situation in the specified regions representing developed area, sub-developed area and less developed area respectively in Guangdong province. Further longitudinal studies in other geographical regions and in different healthcare systems are needed.

\section{Conclusion}

An efficient public health depends on the physical and mental well-being of its medical staff. We found that a significant proportion of medical staff responding to COVID-19 outbreak in
Guangdong Province, China, experienced moderate-severe levels of anxiety and depression during a snapshot in time from early in the pandemic. Perceived stress status and insomnia severity was higher in staff with more risk of infection than those with a less risk, but the prevalence of severe anxiety was similar. We suggest that medical protective equipment and psychological stress assistance should be offered to all medical staff during infectious disease outbreaks, including those indirectly involved. As previously suggested by Chen et al. (2006), a systematic prevention programme that included a series of in-service training, detailed manpower allocation, adequate protective equipment, and the availability of a mental health team is feasible and effective. The current COVID-19 pandemic is unlikely to be the last outbreak of a highly infectious disease and it is important to prepare our health care systems for the future.

Supplementary material. The supplementary material for this article can be found at https://doi.org/10.1017/S0033291720002561.

Financial support. This work was supported by the grants from the Natural Science Foundation of Guangdong Province (2019A 1515011224) and Guangdong Provincial Bureau of Traditional Chinese Medicine (20201008) and the High-level Hospital Construction Project of Guangdong Provincial People's Hospital (DFJH201811 and DFJH201922).

Conflict of interest. The authors declare that they have no competing interests.

\section{References}

Chen, R., Chou, K. R., Huang, Y. J., Wang, T. S., Liu, S. Y., \& Ho, L. Y. (2006). Effects of a SARS prevention programme in Taiwan on nursing staff s anxiety, depression and sleep quality: A longitudinal survey. International Journal of Nursing Studies, 43(2), 215-225. doi: 10.1016/j.ijnurstu.2005.03. 006.

Chen, C. S., Wu, H. Y., Yang, P., \& Yen, C. F. (2005). Psychological distress of nurses in Taiwan who worked during the outbreak of SARS. Psychiatric Services, 56(1), 76. doi: 10.1176/appi.ps.56.1.76.

Chung, K. F., Kan, K. K., \& Yeung, W. F. (2011). Assessing insomnia in adolescents: Comparison of Insomnia Severity Index, Athens Insomnia Scale and Sleep Quality Index. Sleep Medicine, 12(5), 463-470. doi: 10.1016/j.sleep.2010.09.019.

Cohen, S., Kamarck, T., \& Mermelstein, R. (1983). A global measure of perceived stress. Journal of Health and Social Behavior, 24(4), 385-396. doi: $10.2307 / 2136404$.

Cox, R. C., \& Olatunji, B. O. (2020). Sleep in the anxiety-related disorders: A meta-analysis of subjective and objective research. Sleep Medicine Reviews, 51, 101282. doi: 10.1016/j.smrv.2020.101282.

Dyregrov, A., Kristoffersen, J. I., \& Gjestad, R. (1996). Voluntary and professional disaster-workers: Similarities and differences in reactions. Journal of Traumatic Stress, 9(3), 541-555. doi: 10.1007/bf02103663.

Ford, D. E., \& Kamerow, D. B. (1989). Epidemiologic study of sleep disturbances and psychiatric disorders. An opportunity for prevention? JAMA, 262(11), 1479-1484. doi: 10.1001/jama.262.11.1479.

Health Commission. (2020). Outbreak of pneumonia caused by novel coronavirus in Guangdong province. Retrieved from http://wsjkw.gd.gov.cn/gkmlpt/content/2/2881/post_2881518.html\#2571.

Huang, Y., \& Zhao, N. (2020). Mental health burden for the public affected by the COVID-19 outbreak in China: Who will be the high-risk group? Psychology, Health \& Medicine, 20, 1-12. Retrieved from https://doi.org/ 10.1080/13548506.2020.1754438.

Ji, D., Ji, Y. J., Duan, X. Z., Li, W. G., \& Duan, H. J. (2017). Prevalence of psychological symptoms among Ebola survivors and healthcare workers during the 2014-2015 Ebola outbreak in Sierra Leone: A cross-sectional study. Oncotarget, 8(8), 12784-12791. doi: 10.18632/oncotarget.14498.

Kendler, K. S., Thornton, L. M., \& Gardner, C. O. (2000). Stressful life events and previous episodes in the etiology of major depression in women: An 
evaluation of the 'kindling' hypothesis. The American Journal of Psychiatry, 157(8), 1243-1251. doi: 10.1176/appi.ajp.157.8.1243.

Khatony, A., Zakiei, A., Khazaie, H., Rezaei, M., \& Janatolmakan, M. (2020). International nursing: A study of sleep quality among nurses and its correlation with cognitive factors. Nursing Administration Quarterly, 44(1), E1E10. doi: $10.1097 /$ naq. 0000000000000397.

Lai, J., Ma, S., Wang, Y., Cai, Z., Hu, J., Wei, N., ... Hu, S. (2020). Factors associated with mental health outcomes among health care workers exposed to coronavirus disease 2019. JAMA Network Open, 3(3), e203976. doi: 10.1001/jamanetworkopen.2020.3976.

Lancet (2020). Protecting Chinese doctors. Lancet (London, England), 395 (10218), 90. doi: 10.1016/s0140-6736(20)30003-9.

Lehmann, M., Bruenahl, C. A., Addo, M. M., Becker, S., Schmiedel, S., Lohse, A. W., ... Löwe, B. (2016). Acute Ebola virus disease patient treatment and health-related quality of life in health care professionals: A controlled study. Journal of Psychosomatic Research, 83, 69-74. doi: 10.1016/j.jpsychores.2015. 09.002.

Leung, D. Y., Lam, T. H., \& Chan, S. S. (2010). Three versions of Perceived Stress Scale: Validation in a sample of Chinese cardiac patients who smoke. BMC Public Health, 10, 513. doi: 10.1186/1471-2458-10-513.

Li, Q., Guan, X., Wu, P., Wang, X., Zhou, L., Tong, Y., ... Feng, Z. (2020). Early transmission dynamics in Wuhan, China, of novel coronavirus-infected pneumonia. The New England Journal of Medicine, 382(13), 1199-1207. doi: 10.1056/NEJMoa 2001316.

Lin, L., \& Li, T. S. (2020). Interpretation of 'guidelines for the diagnosis and treatment of novel coronavirus (2019-nCoV) infection by the National Health Commission (Trial Version 5)'. Zhonghua yi xue za zhi, 100, E001. doi: 10.3760/cma.j.issn.0376-2491.2020.0001.

Lu, W., Wang, H., Lin, Y., \& Li, L. (2020). Psychological status of medical workforce during the COVID-19 pandemic: A cross-sectional study. Psychiatry Research, 288, 112936. doi: 10.1016/j.psychres.2020.112936.

Matsuishi, K., Kawazoe, A., Imai, H., Ito, A., Mouri, K., Kitamura, N., ... Mita, T. (2012). Psychological impact of the pandemic (H1N1) 2009 on general hospital workers in Kobe. Psychiatry and Clinical Neurosciences, 66(4), 353-360. doi: 10.1111/j.1440-1819.2012.02336.x.

Maunder, R. (2004). The experience of the 2003 SARS outbreak as a traumatic stress among frontline healthcare workers in Toronto: Lessons learned. Philosophical Transactions of the Royal Society of London. Series B, Biological Sciences, 359(1447), 1117-1125. doi: 10.1098/rstb.2004.1483.

Maunder, R., Hunter, J., Vincent, L., Bennett, J., \& Mazzulli, T. (2003). The immediate psychological and occupational impact of the 2003 SARS outbreak in a teaching hospital. Canadian Medical Association Journal, 168 (10), 1245-1251. doi: 10.1001/jama.289.18.2432.

Maunder, R. G., Lancee, W. J., Rourke, S., Hunter, J. J., Goldbloom, D., Balderson, K., ... Fones, C. S. (2004). Factors associated with the psychological impact of severe acute respiratory syndrome on nurses and other hospital workers in Toronto. Psychosomatic Medicine, 66(6), 938-942. doi: 10.1097/01.psy.0000145673.84698.18.

Meerlo, P., Sgoifo, A., \& Suchecki, D. (2008). Restricted and disrupted sleep: Effects on autonomic function, neuroendocrine stress systems and stress responsivity. Sleep Medicine Reviews, 12(3), 197-210. doi: 10.1016/ j.smrv.2007.07.007.

Morin, C. M., Beaulieu-Bonneau, S., LeBlanc, M., \& Savard, J. (2005). Self-help treatment for insomnia: A randomized controlled trial. Sleep, 28(10), 13191327. doi: $10.1093 /$ sleep/28.10.1319.

National Health Commission. (2020). National Health Commission's briefing on the pneumonia epidemic situation (in Chinese). Retrieved from http:// www.nhc.gov.cn/.

Shigemura, J., Tanigawa, T. I., \& Nomura, S. (2012). Psychological distress in workers at the Fukushima nuclear power plants. JAMA, 308(7), 667-669. doi: 10.1001/jama.2012.9699.

Tang, Y. F., Liu, Y., Gao, C. H., Song, J., Wang, R., Li, Z. Y., ... Liao, P. H. (2020). Symptom cluster of ICU nurses treating COVID-19 pneumonia patients in Wuhan, China. Journal of Pain and Symptom Management. doi: 10.1016/j.jpainsymman.2020.03.039

Weinberg, A., \& Creed, F. (2000). Stress and psychiatric disorder in healthcare professionals and hospital staff. Lancet (London, England), 355(9203), 533 537. doi: 10.1016/s0140-6736(99)07366-3.

Weisæth, L. (2007). A study of behavioural response to an industrial disaster. Acta Psychiatrica Scandinavica, 80(Suppl. 355), 13-24. doi: 10.1111/ j.1600-0447.1989.tb05250.x.

WHO. (2020a). Statement on the second meeting of the International Health Regulations (2005) Emergency Committee regarding the outbreak of novel coronavirus (2019-nCoV). Retrieved from https://www.who.int/news-room/ detail/30-01-2020-statement-on-the-second-meeting-of-the-internationalhealth-regulations-(2005)-emergency-committee-regarding-the-outbreak-ofnovel-coronavirus-(2019-ncov).

WHO. (2020b). Novel Coronavirus (2019-nCoV) Situation Report - 12. Retrieved from https://www.who.int/docs/default-source/coronaviruse/ situation-reports/20200201-sitrep-12-ncov.pdf?sfvrsn=273c5d35_2.

WHO. (2020c). Coronavirus. Retrieved from https://www.who.int/emergencies/ diseases/novel-coronavirus-2019.

Wong, S. Y., Zhang, D. X., Li, C. C., Yip, B. H., Chan, D. C., Ling, Y. M., ... Wing, Y. K. (2017). Comparing the effects of mindfulness-based cognitive therapy and sleep psycho-education with exercise on chronic insomnia: A randomised controlled trial. Psychotherapy and Psychosomatics, 86(4), 241-253. doi: 10.1159/000470847.

Wu, P., Fang, Y., Guan, Z., Fan, B., Kong, J., Yao, Z., ... Hoven, C. W. (2009). The psychological impact of the SARS epidemic on hospital employees in China: Exposure, risk perception, and altruistic acceptance of risk Canadian Journal of Psychiatry. Revue Canadienne de Psychiatrie, 54(5), 302-311. doi: 10.1177/070674370905400504.

Xiao, H., Zhang, Y., Kong, D., Li, S., \& Yang, N. (2020). The effects of social support on sleep quality of medical staff treating patients with coronavirus disease 2019 (COVID-19) in January and February 2020 in China. Medical Science Monitor: International Medical Journal of Experimental and Clinical Research, 26, e923549. doi: 10.12659/msm.923549.

Zigmond, A. S., \& Snaith, R. P. (1983). The hospital anxiety and depression scale. Acta Psychiatrica Scandinavica, 67(6), 361-370. doi: 10.1111/ j.1600-0447.1983.tb09716.x. 